\title{
Nascent-peptide-mediated ribosome stalling at a stop codon induces mRNA cleavage resulting in nonstop mRNA that is recognized by tmRNA
}

\author{
TAKAFUMI SUNOHARA, KAORU JOJIMA, YASUFUMI YAMAMOTO, TOSHIFUMI INADA, and HIROJI AIBA \\ Division of Biological Science, Graduate School of Science, Nagoya University, Chikusa, Nagoya 464-8602, Japan
}

\begin{abstract}
Recent studies have established that tmRNA-mediated protein tagging occurs at stop codons depending on the C-terminal amino acid sequence of the nascent polypeptide immediately adjacent to those codons. We investigate here how the trans-translation at a stop codon occurs by using model crp genes encoding variants of cAMP receptor protein (CRP). We demonstrate that a truncated crp mRNA is efficiently produced along with a normal transcript from the model gene where tmRNA-mediated protein tagging occurs. The truncated $\operatorname{crp}$ mRNA was not detected in the presence of tmRNA, indicating that its degradation was facilitated by tmRNA. The major 3 '-ends of the truncated $\operatorname{crp}$ mRNA in cells unable to express tmRNA were mapped at and near the stop codon. When RNA derived from the model crp-crr fusion gene was analyzed, crr mRNA was detected as a downstream cleavage product along with the upstream $\operatorname{crp}$ mRNA. These results are compatible with the hypothesis that ribosome stalling caused by the tagging-provoking sequences leads to endonucleolytic cleavage of mRNA around the stop codon, resulting in nonstop mRNA. In addition, the data are consistent with the view that mRNA cleavage is the cause of trans-translation at stop codons. Neither the bacterial toxin RelE nor the known major endoribonucleases are required for this cleavage, indicating that either other endoribonuclease(s) or the ribosome itself would be responsible for the mRNA cleavage in response to ribosome stalling caused by the particular nascent peptides.
\end{abstract}

Keywords: tmRNA; stop codon; ribosome stalling; mRNA cleavage; nascent peptide

\section{INTRODUCTION}

The bacterial tmRNA, also called SsrA RNA, is a unique molecule that has properties of both tRNA and mRNA (Keiler et al. 1996; Karzai et al. 2000). When a ribosome stalls on a problematic mRNA, typically at the 3 '-end of a truncated mRNA without an in-frame stop codon, tmRNA is recruited to the ribosome, in which it acts first as an alanyl-tRNA and then as an mRNA to direct the addition of a short peptide tail to the polypeptide. This cotranslation reaction (trans-translation) terminates at the stop codon that follows the tmRNA reading frame, releasing both the ribosome and the tagged polypeptide. The tagged polypeptide is recognized and degraded by several ATP-dependent proteases. In addition, tmRNA-mediated trans-translation has been shown to facilitate the degradation of truncated

Reprint requests to: Hiroji Aiba, Division of Biological Science, Graduate School of Science, Nagoya University, Chikusa, Nagoya 464-8602, Japan; e-mail: i45346a@nucc.cc.nagoya-u.ac.jp; fax: 81-52-789-3001.

Article and publication are at http://www.rnajournal.org/cgi/doi/ 10.1261/rna.5169404.
mRNAs by removing stalled ribosomes and thus allowing $3^{\prime}$-to-5' exonucleases to access the free mRNA 3'-end (Yamamoto et al. 2003). Thus, the quality-control function of the tmRNA system is more elaborate than originally thought because it not only degrades aberrant polypeptides but also prevents production of aberrant polypeptides through the rapid elimination of damaged mRNAs.

The well-known target for the tmRNA system is the $3^{\prime}$ end of a truncated mRNA lacking an in-frame stop codon where the ribosome is expected to stall (Keiler et al. 1996). The truncated "nonstop" mRNAs derived from natural genes are generated in cells either by incomplete transcription (Abo et al. 2000) or by nuclease cleavages of an mRNA (Yamamoto et al. 2003). A ribosome also reaches the $3^{\prime}$-end of an mRNA when a normal stop codon is erroneously translated either in the presence of nonsense suppressor tRNAs (Ueda et al. 2002) or in the presence of misreading drugs (Abo et al. 2002). The tmRNA system appears to act also at a run of rare codons on an mRNA where ribosomes are expected to stall because of a deficiency of cognate aminoacyl-tRNAs (Roche and Sauer 1999). In addition, trans- 
translation could occur at a position corresponding to the normal termination codon in certain conditions, depending on the presence of rare arginine codons near the adjacent inefficient UGA termination codon (Collier et al. 2002; Hayes et al. 2002b) or the amino acid sequence of the nascent polypeptide prior to stop codons (Hayes et al. 2002a; Sunohara et al. 2002).

In principle, there are two different mechanisms by which trans-translation occurs at a stop codon. Certain nascent peptides may cause ribosome stalling by partially preventing the action of release factors (RFs) without damaging mRNA itself. In this case, tmRNA may enter the A-site by competing with RFs or near-cognate aminoacyl-tRNAs. An alternative possibility is that the ribosome stalling somehow induces endonucleolytic cleavage of the mRNA at or prior to the stop codon, resulting in "nonstop" mRNAs. In the latter case, there is no competition between tmRNA and RFs because the A-site is empty. Recent findings that ribosome stalling may cause mRNA cleavages in several cases either in vivo (Loomis et al. 2001; Drider et al. 2002) or in vitro (Pedersen et al. 2003) have prompted us to examine whether the trans-translation at the stop codons is associated with mRNA cleavage or not. We demonstrate here that truncated crp mRNAs encoding cAMP receptor protein (CRP) are efficiently generated in cells lacking tmRNA from a model crp gene in which the stop-codon-dependent tagging of CRP occurs. The truncated crp mRNA is rapidly degraded in the presence of tmRNA. The 3 '-ends of the truncated $c r p$ mRNA are mapped around the stop codon. We propose that ribosome stalling caused by certain nascent peptides leads to endonucleolytic cleavages around the A-site of the stalled ribosome, resulting in nonstop mRNA, a typical substrate for trans-translation.

\section{RESULTS}

\section{Effects of the identity of -2 residues on tagging of CRP-XP}

Particular C-terminal sequences of the nascent peptides, such as the LESG tetrapeptide and a subset of XP dipeptides, could cause efficient tmRNA-mediated trans-translation at stop codons, resulting in tagging of full-length proteins (Hayes et al. 2002a; Sunohara et al. 2002). It was reported that high levels of tagging of YbeL ending with proline were observed when the penultimate $(-2)$ residue was D, E, I, V, or P (Hayes et al. 2002a). To investigate further how the nature of $\mathrm{C}$-terminal amino acid residues affects the tagging at stop codons, we also examined systematically the effects of the -2 residues on tagging of CRP$\mathrm{XP}$ proteins. A series of CRP-XP proteins (Fig. 1) was coexpressed with a mutant tmRNA-DD encoding a proteaseresistant tag sequence and analyzed by Western blotting using anti-CRP antibodies. As shown in Figure 2, CRP-XP proteins were efficiently tagged when $\mathrm{X}$ was $\mathrm{D}, \mathrm{F}, \mathrm{G}$, or $\mathrm{P}$,
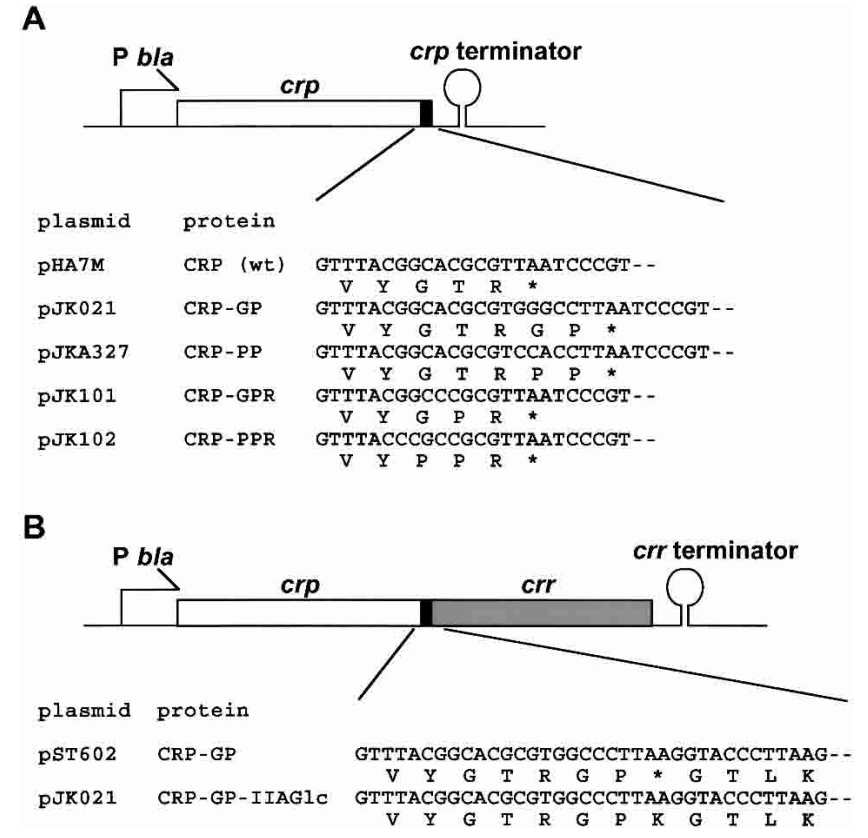

FIGURE 1. Schematic drawing of the $(A)$ crp and $(B)$ crp-crr fusion genes used in this study. The open and shaded rectangles represent the coding region for CRP and IIA ${ }^{\text {Glc }}$, respectively. The black box represents the altered 3 '-portion of the CRP coding region. The nucleotide sequence and amino acid sequence (in one-letter symbols) of the variable region are shown below the diagram.

whereas no or only weak tagging was observed for proteins containing other amino acid residues at the -2 position (Fig. 2). Thus, the tagging spectrum of CRP-XP proteins significantly deviates from that of YbeL-XP proteins (Hayes et al. 2002a). This implies that the nature of amino acid residues upstream of the -2 position also affects the tmRNA-mediated tagging of proteins ending with a proline residue.

\section{Identification of the tagging site}

The untagged and tagged CRP-GP proteins were purified from $\Delta$ crp $s s r A^{D D}$ cells carrying pJK021. The purified pro-

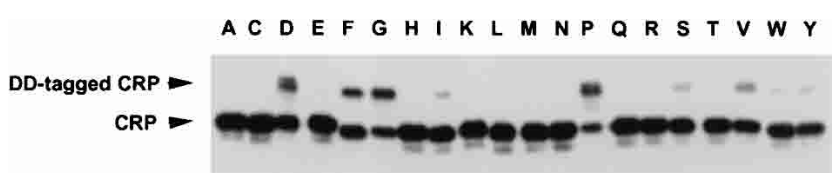

FIGURE 2. Tagging of a series of CRP-XP proteins. Lysates equivalent to $\mathrm{OD}_{600}=0.005$ unit prepared from TA481 $\left(\Delta \operatorname{crp} s s r A^{D D}\right)$ cells harboring pHA7 derivatives carrying altered $c r p$ genes that encode CRP-XP proteins were analyzed by Western blotting using anti-CRP antibodies. The amino acid residues at the -2 position are indicated by a one-letter symbol. The -2 and -1 codons were (A) GCG CCG; (C) TGC CCG; (D) GAT CCA; (E) GAA CCG; (F) TTC CCC; (G) GGC CCT; (H) CAT CCG; (I) ATT CCG; (K) AAA CCG; (L) CTG CCG; (M) ATG CCG; (N) AAC CCG; (P) CCA CCT; (Q) CAG CCG; (R) CGC CCG; (S) AGC CCG; (T) ACC CCG; (V) GTG CCT; (W) TGG CCG; and (Y) TAT CCG. 


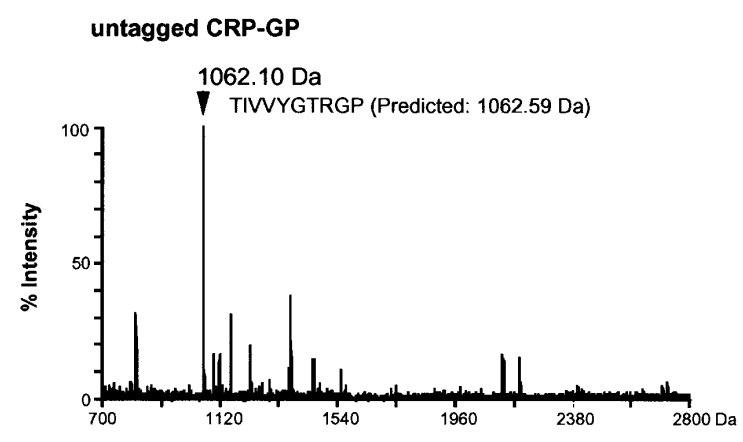

tagged CRP-GP

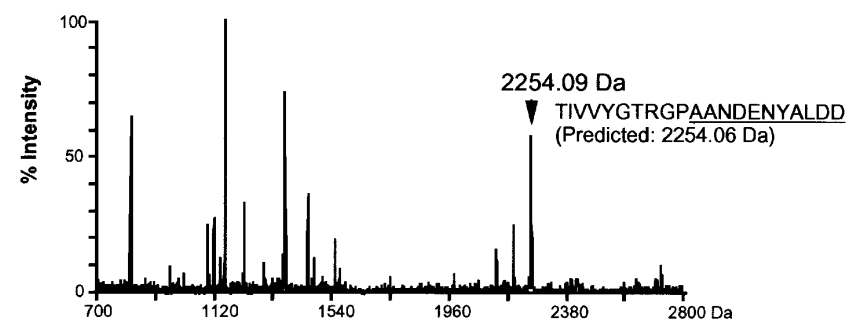

FIGURE 3. Mass spectrometry analysis of untagged and tagged CRPGP. Purified proteins were separated on a $12 \%$ SDS-polyacrylamide gel electrophoresis followed by Coomassie Brilliant Blue staining. The bands corresponding to untagged and tagged CRP-GP were cut out from the gel. The gel was treated with lysyl endopeptidase and subjected to mass spectrometry analysis. The signals that are expected to correspond to the $\mathrm{C}$-terminal fragments are shown by arrowheads along with the observed mass. The expected peptide sequences and molecular weights of the C-terminal fragments of untagged (upper) and tagged CRP-GP (lower) generated by lysyl endopeptidase digestion are shown below the observed mass.

teins were subjected to SDS-polyacrylamide gel electrophoresis followed by Coomassie Brilliant Blue staining. The polypeptide bands corresponding to the untagged and DDtagged proteins were excised from the gel and digested in gel with lysyl endopeptidase, which specifically cleaves the peptide bond after lysine residues. The eluted peptides were analyzed by MALDI-TOF mass spectrometry. The peptidase digestion of the untagged band gave a signal with a mass of 1062.10 D, which corresponds to that expected for the Cterminal fragment size of CRP-GP along with several other signals (Fig. 3, upper). When the lysyl endopeptidase digest of the tagged band was analyzed, the 1062.10-D signal was no longer observed, and a new signal with a mass of 2254.09 $\mathrm{D}$, which corresponds to a junction peptide containing the C-terminal fragment of CRP-GP plus the tag, appeared (Fig. 3, lower). All other signals observed in the digest of the untagged band were also detected in the digest of the tagged band. These results clearly indicate that the stop-codondependent tmRNA tagging of CRP-GP occurs predominantly just after the last $\mathrm{C}$-terminal residue as in the case of CRP-LESG (Sunohara et al. 2002).

\section{The tagging-provoking sequences are effective specifically at stop codons}

We showed previously that the presence of even one amino acid residue between a tagging-provoking tetrapeptide LESG and the stop codon eliminates the tagging of the full-length proteins (Sunohara et al. 2002). To test whether this is also the case for XP dipeptides, the C-terminal -3 and -2 residues of the wild-type CRP were converted to GP or PP, and the expression of CRP-GPR and CRP-PPR was analyzed in three isogenic strains regarding the $s s r A$ allele. No tagging was observed in these altered CRP proteins (Fig. 4, lanes 10-15) as in the case of wild-type CRP (Fig. 4, lanes $1-3)$, whereas efficient tagging was detected again in CRPGP and CRP-PP (Fig. 4, lanes 4-9). Thus, the taggingprovoking sequences seem to induce trans-translation specifically at stop codons, although we do not exclude a possibility that they induce trans-translation at certain sense codons depending on the surrounding sequences. It should be noted that the tagging-provoking sequences significantly reduce the level of CRP in the presence of wild-type tmRNA through efficient degradation of the tagged proteins (Fig. 4, lanes 5,8).

\section{Truncated $\operatorname{crp}$ mRNA is produced}

The trans-translation at stop codons could occur through the recruitment of tmRNA at an internal mRNA site within a stalled ribosome. Alternatively, it can be simply explained if a truncated mRNA lacking a stop codon is produced for some reason in response to ribosome stalling at the stop codon. To test whether this is the case or not, total RNA was prepared from cells carrying one of several plasmid-borne variant crp genes both in the presence and absence of tmRNA and was analyzed by Northern blotting using a DNA probe specific to the $\operatorname{crp}$ mRNA. When the wild-type CRP was expressed, a normal crp mRNA of $\sim 700$ nucleotides (nt) was detected both in the presence and the absence of tmRNA (Fig. 5, lanes 1,2). Interestingly, another shorter band appeared, resulting in a significant reduction in the amount of full-length crp mRNA when CRP-GP and CRP-PP were expressed in the absence of tmRNA (Fig. 5, lanes 3,5). The

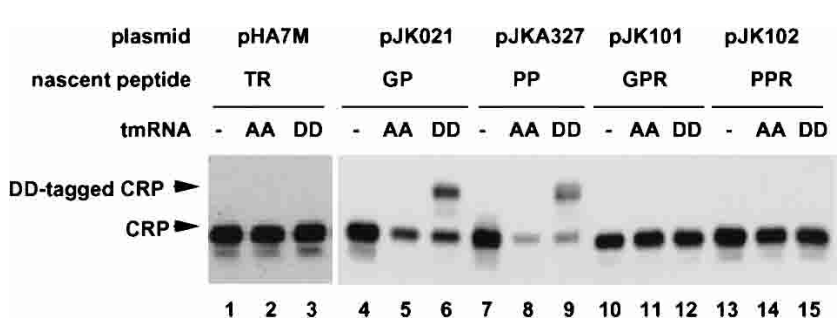

FIGURE 4. Positional effect of the tagging-provoking sequences. Lysates equivalent to $\mathrm{OD}_{600}=0.005$ unit prepared from TA341 $(\Delta c r p$ $\left.s s r A^{+}\right)$, TA501 $(\Delta c r p \Delta s s r A)$, or TA481 $\left(\Delta c r p s s r A^{D D}\right)$ cells harboring indicated plasmids were analyzed by Western blotting using anti-CRP antibodies. 


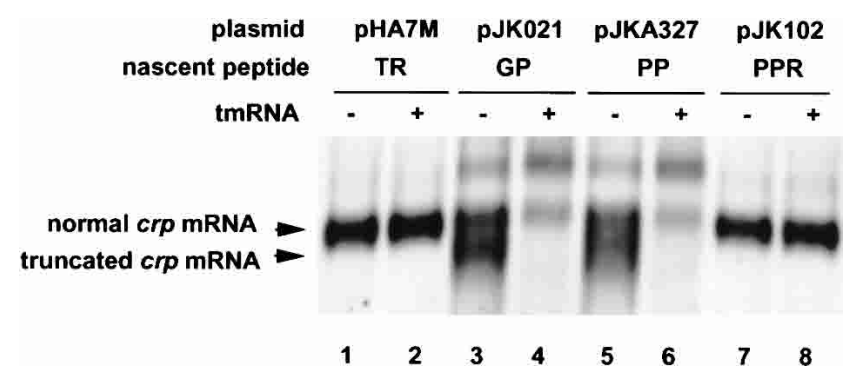

FIGURE 5. Northern blot analysis of $c r p$ mRNAs derived from the $c r p$ genes encoding CRP variants. Total RNA was prepared from TA341 $\left(\Delta c r p s s A^{+}\right)$and TA501 $(\Delta c r p \Delta s s r A)$ cells harboring indicated plasmids. Either $0.15 \mu \mathrm{g}$ (lanes $1,2,3,5,7,8)$ or $0.5 \mu \mathrm{g}$ (lanes 4,6 ) of RNA was resolved by electrophoresis on a $2.0 \%$ agarose-formaldehyde gel. Northern blot analysis was performed using the DIG-labeled crp probe. RNA bands corresponding to the full-length and truncated crp mRNAs are indicated by arrowheads.

shorter crp mRNA was no longer observed in the presence of tmRNA despite the use of threefold more RNA (Fig. 5, lanes 4,6). Essentially the same results were obtained when RNA from genes encoding CRP-DP and CRP-FP was analyzed (data not shown). When the tagging-negative CRPPPR was expressed, only the full-length $\operatorname{crp}$ mRNA was detected (Fig. 5, lanes 7,8). Thus, the tmRNA-mediated tagging of CRP at stop codons is tightly associated with the production of the shorter truncated crp mRNA. These results indicate that the truncated $c r p$ mRNA is generated as a result of ribosome stalling at stop codons, which in turn would be recognized by tmRNA. We found previously that the truncated mRNAs are released from the stalled ribosome and rapidly degraded during trans-translation (Yamamoto et al. 2003). This is why the truncated crp mRNA was not detected in the presence of tmRNA.

\section{The truncated $c r p$ mRNA lacks a stop codon}

To determine the sequence of the $3^{\prime}$-end of the truncated crp mRNA, total RNA prepared from tmRNA-deficient cells expressing CRP-GP was hybridized with a DNA probe C, ${ }^{32} \mathrm{P}$-labeled at its $3^{\prime}$-end. DNA probe $\mathrm{C}$ covers the $3^{\prime}$-region of the $\operatorname{crp}$ gene including a part of the coding sequence and the terminator sequence. The hybrids were treated with S1 nuclease, and the products were analyzed by electrophoresis on a sequencing gel. As shown in Figure 6, two clusters of S1-resistant bands (referred to as I and II) were detected. Cluster I represents the full-length $\operatorname{crp}$ mRNA, and its major $3^{\prime}$-ends were mapped just after the inverted repeat sequence of the crp terminator as previously shown (Abe et al. 1999). Cluster II corresponds to the truncated $\operatorname{crp}$ mRNA, and its major $3^{\prime}$-ends were mapped at the stop codon. Thus, the truncated $\operatorname{crp}$ mRNA apparently lacks a stop codon.

\section{Nonstop mRNA is generated by endonucleolytic cleavage}

Several possibilities could be considered for the production of the truncated $\operatorname{crp}$ mRNA. First, it can be produced by premature termination of transcription. This possibility is less likely because the nucleotide sequence corresponding to the tagging-provoking peptides has no specific features for transcription terminator. In fact, the production of truncated $c r p$ mRNA was markedly reduced when translation was prevented by chloramphenicol (data not shown), indicating that translational pausing rather than transcriptional pausing is responsible for the formation of truncated $c r p$ mRNA. Second, 3'-to-5' exonucleolytic trimming of the mature transcript up to the stalled ribosome would account for the generation of the truncated $c r p$ mRNA. This second possibility is also less likely, although it cannot be ruled out because the mature crp mRNA posseses the $3^{\prime}$ terminator hairpin structure that can act as a barrier of $3^{\prime}$-to- $5^{\prime}$ exo-

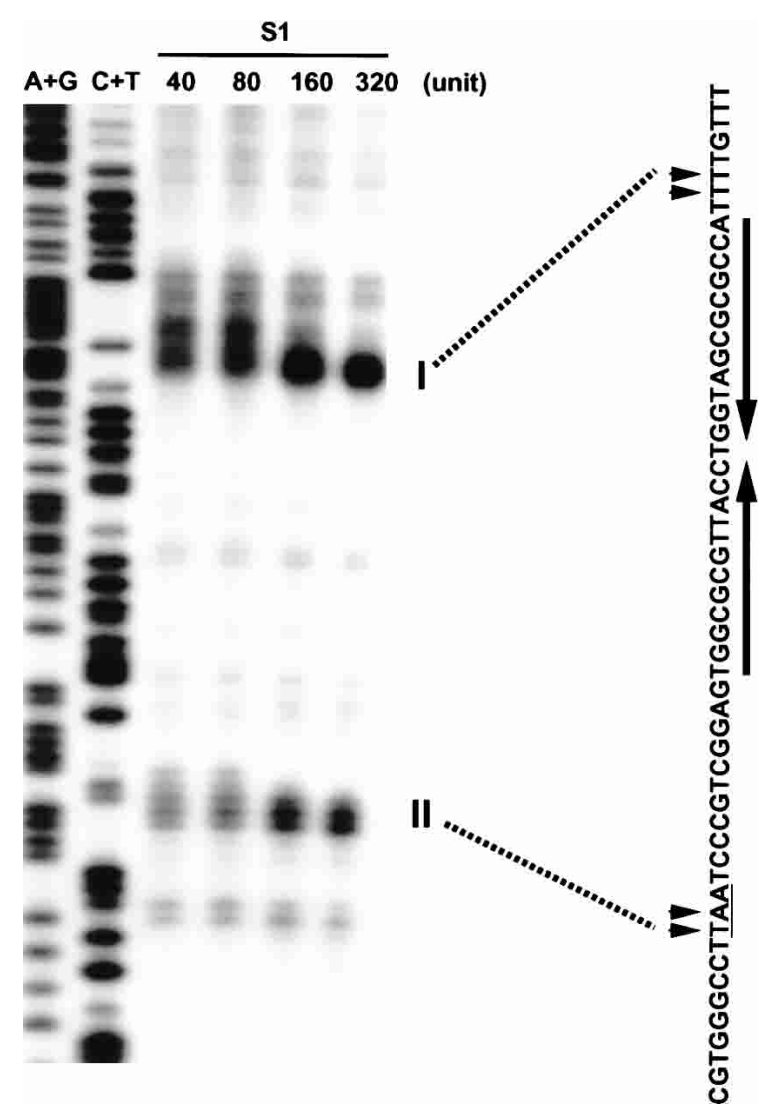

FIGURE 6. Determination of $3^{\prime}$-ends of the crp mRNAs. Total RNA $(50 \mu \mathrm{g})$ prepared from TA501 $(\Delta c r p \Delta s s r A)$ harboring pJK021 was hybridized with the DNA probe $\mathrm{C}^{32} \mathrm{P}$-labeled at its $3^{\prime}$-end of the template strand, and the hybrids were treated with the indicated amounts of S1 nuclease. The products were dissolved in $20 \mu \mathrm{L}$ of loading buffer ( $8 \mathrm{M}$ urea, $0.025 \%$ bromophenol blue, $0.025 \%$ xylene cyanol, $90 \mathrm{mM}$ Tris-borate at $\mathrm{pH} 8.3$, and $1 \mathrm{mM}$ EDTA), and $2 \mu \mathrm{L}$ of each sample was analyzed on an $8 \%$ polyacrylamide- $8 \mathrm{M}$ urea gel along with products of an $A+G$ and $C+T$ chemical sequencing reaction of the fragment. Cluster I represents the 3 '-ends of the normal crp mRNA, whereas cluster II corresponds to the truncated crp mRNA. The nucleotide sequence around the stop codon of the altered crp gene is shown on the right. The arrowheads indicate the major $3^{\prime}$-ends identified by the $\mathrm{S} 1$ analysis. The TAA stop codon is underlined. The GC-rich inverted repeat sequence of the terminator is indicated by vertical arrows. 
nucleolytic attack. The most likely mechanism for the generation of truncated crp mRNA is endonucleolytic cleavage of the longer transcript in response to ribosome stalling. If the endonucleolytic cleavage model is correct, the downstream mRNA is expected to be produced along with the upstream crp mRNA as a cleavage product. To facilitate the detection of this presumptive downstream cleavage product, we constructed the crp-TAA-crr fusion gene (pST602) in which the IIA ${ }^{\text {Glc }}$ ORF encoded by $c r r$ was fused just after the TAA codon of a variant crp gene encoding CRP-GP. The crp-AAA-crr fusion gene (pJK107) was also constructed as a control in which the TAA codon for CRP-GP was replaced by AAA. This control gene encodes a CRP-GP-IIA ${ }^{\text {Glc }}$ fusion protein. As expected, the tmRNA-mediated tagging and proteolysis of CRP-GP was observed in the crp-TAA-crr fusion gene, and the CRP-GP-IIA ${ }^{\text {Glc }}$ fusion protein was expressed without tagging in the crp-AAA-crr fusion gene (Fig. 7A). Northern blot analysis using a $c r p$ DNA probe revealed that the upstream crp mRNA of $650 \mathrm{nt}$ was produced along with the crp-crr mRNA of $1200 \mathrm{nt}$ from pST602 in the absence of tmRNA (Fig. 7B, lane 1). The crp mRNA was no longer observed in the presence of tmRNA, indicating again that it is very unstable (Fig. 7B, lane 2). When Northern blot analysis was performed by using a $\mathrm{crr}$ DNA probe, an RNA band of $\sim 550 \mathrm{nt}$ corresponding to the downstream $\mathrm{cr}$ mRNA was clearly detected along with the crp-crr mRNA in the absence of tmRNA (Fig. 7C, lane 1). In the presence of tmRNA, the abundance of the $\mathrm{crr}$ band was increased, whereas the level of the full-length $\mathrm{crp}-\mathrm{crr}$ mRNA was reduced (Fig. 7C, lane 2). The reduction of the full-length $c r p-c r r$ mRNA in the presence of tmRNA was also observed when the crp DNA probe was used (Fig. 7B). These data are consistent with a view that endonucleolytic cleavage of the full-length $c r p-c r r$ mRNA occurs, resulting in both $\mathrm{crp}$ and $\mathrm{crr}$ mRNAs in response to the ribosome stalling. The increase of the downstream cleavage product, crr mRNA, and the decrease of the full-length $\mathrm{crp}-\mathrm{crr}$ mRNA, in the presence of tmRNA indicates that the endonucleolytic cleavage occurs more efficiently in the presence of tmRNA. It should be noted that the abundance of the downstream $\mathrm{crr}$ mRNA relative to the full-length $c r p-c r r$ mRNA was significantly lower than that of the upstream crp mRNA. This indicates that the downstream crr mRNA generated by endonucleolytic cleavage may be less stable compared with the upstream $c r p$ mRNA in the absence of tmRNA. The specific truncated $c r r$ and $c r p$ mRNAs were no longer produced when the stop codon of $\operatorname{crp}$ was replaced by a sense codon (Fig. 7B,C, lanes 3,4).

\section{Bacterial toxin RelE is not required for the mRNA cleavage}

A bacterial toxin RelE was found to induce endonucleolytic cleavage of mRNAs bound to ribosomes in vitro at specific sites including stop codons in response to a stalled ribosome
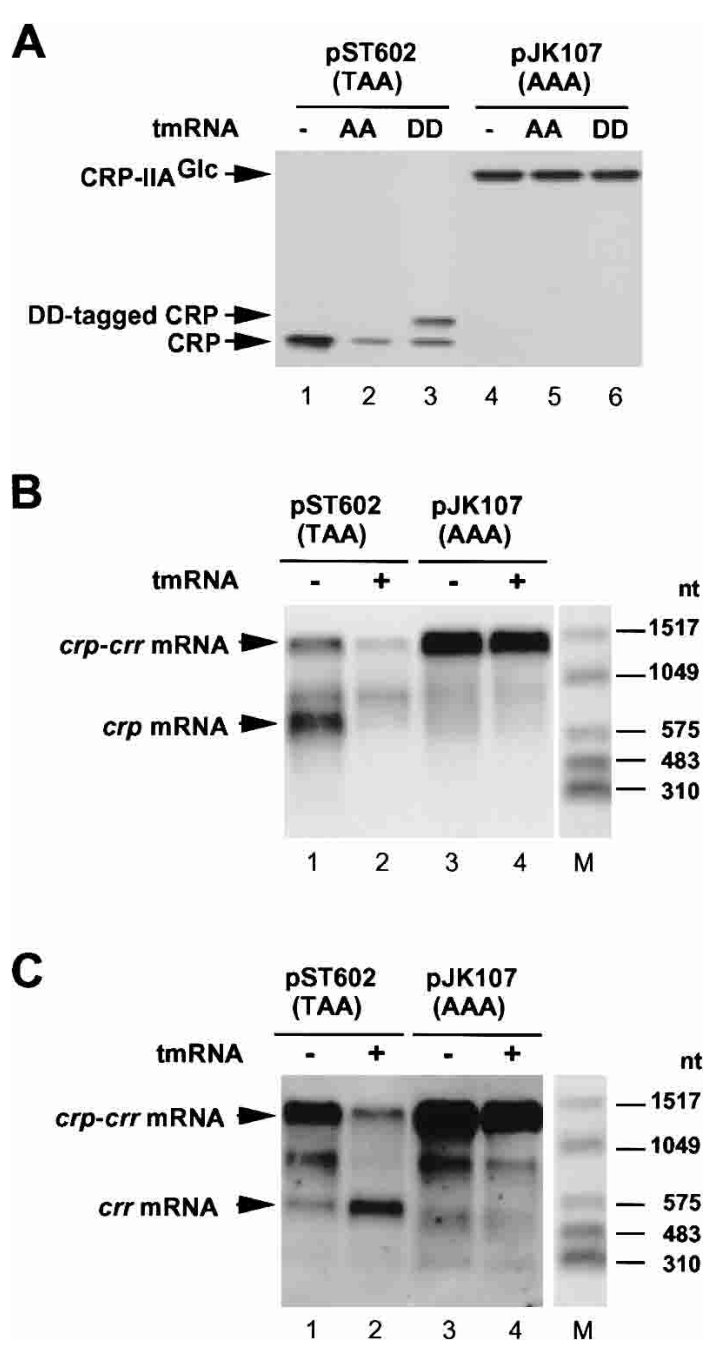

FIGURE 7. Analyses of protein and RNA derived from the crp-crr fusion genes. (A) Western blot analysis of CRP proteins. Lysates equivalent to $\mathrm{OD}_{600}=0.005$ unit prepared from TA341 $\left(\Delta c r p s s r A^{+}\right)$, TA501 $(\Delta c r p \Delta s s r A)$, or TA481 $\left(\Delta c r p s s r A^{D D}\right)$ cells harboring pST602 (lanes 1-3) and pJK107 (lanes 4-6) were analyzed by Western blotting using anti-CRP antibodies. (B) Northern blot analysis of $\operatorname{crp}$ mRNAs derived from the $c r p-c r r$ fusion genes. Total RNA $(1 \mu \mathrm{g})$ prepared from TA341 $\left(\Delta c r p s s r A^{+}\right)$and TA501 $(\Delta c r p \Delta s s r)$ cells harboring pST602 (lanes 1,2 ) and pJK107 (lanes 3,4 ) were resolved by electrophoresis on a $1.5 \%$ agarose-formaldehyde gel. Northern blot analysis was performed using the DIG-labeled crp probe. RNA bands corresponding to the full-length $c r p-c r r$ and truncated $c r p$ mRNAs are indicated by arrowheads. (Lane M) RNA size markers. (C) Northern blot analysis of $\mathrm{cr}$ mRNAs derived from the crp-crr fusion genes. Total RNA $(1 \mu \mathrm{g})$ prepared from TA341 $\left(\Delta c r p s s r A^{+}\right)$and TA501 $(\Delta c r p$ $\Delta s s r A$ ) cells harboring pST602 (lanes 1,2) and pJK107 (lanes 3,4) were resolved by electrophoresis on a $1.5 \%$ agarose-formaldehyde gel. Northern blot analysis was performed using the DIG-labeled crr probe. RNA bands corresponding to the full-length $c r p-c r r$ and truncated $c r r$ mRNAs are shown by arrowheads. (Lane M) RNA size markers.

(Pedersen et al. 2003). More recently, it has been demonstrated that either overproduction of RelE or amino acid starvation could induce endonucleolytic mRNA cleavage in vivo (Christensen and Gerdes 2003). To examine whether RelE is involved or not in the endonucleolytic cleavage of 
mRNA around the stop codon in the model crp genes, we disrupted the entire relBE region in both $s s r A^{+}$and $s s r A^{-}$ strains, and plasmid pJK021 was introduced in these strains. The effects of the relE disruption on the expression of the fusion was analyzed by Northern blotting using both crp (Fig. 8A) and $\mathrm{crr}$ probes (Fig. 8B). The truncated mRNAs were generated "normally" as in the case of the relBE strain. These data indicate that RelE is not required for the generation of truncated $c r p$ mRNA in response to the stalled ribosome caused by tagging-provoking sequences prior to stop codons in our system.

\section{DISCUSSION}

A well-known target for the tmRNA system is the $3^{\prime}$-end of a truncated mRNA lacking an in-frame stop codon where the ribosome stalling is expected to occur because of the lack of the normal translation termination signal (Keiler et al. 1996). In this case, tmRNA-mediated trans-translation

A

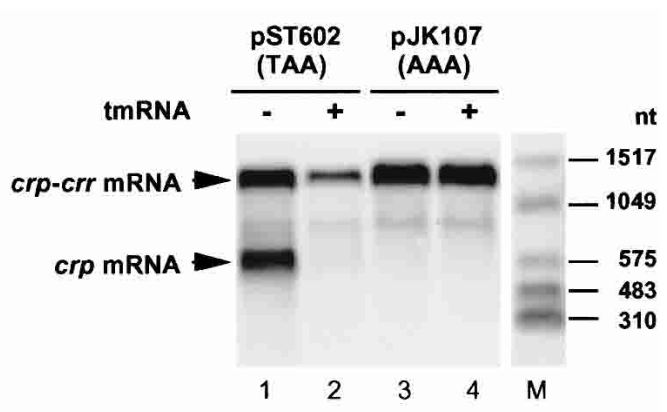

B

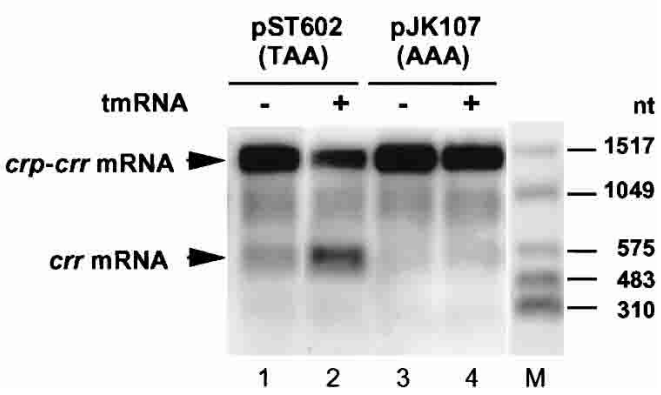

FIGURE 8. Effect of relEB disruption on mRNA cleavage. (A) Northern blot analysis of $c r p$ mRNAs derived from the crp-crr fusion genes. Total RNA $(1 \mu \mathrm{g})$ prepared from ST100 $(\Delta$ relEB $)$ and ST101 $(\Delta s s r A$ $\Delta$ relEB) cells harboring pST602 (lanes 1,2 ) and pJK107 (lanes 3,4) were resolved by electrophoresis on a $1.5 \%$ agarose-formaldehyde gel. Northern blot analysis was performed using the DIG-labeled crp probe. RNA bands corresponding to the full-length $\mathrm{crp}-\mathrm{crr}$ and truncated $\operatorname{crp}$ mRNAs are indicated by arrowheads. (Lane M) RNA size markers. (B) Northern blot analysis of $\mathrm{crr}$ mRNAs derived from the $c r p-c r r$ fusion genes. Total RNA $(1 \mu \mathrm{g})$ prepared from ST100 $(\Delta r e l E B)$ and ST101 ( $\Delta$ ssrA $\Delta$ relEB) cells harboring pST602 (lanes 1,2 ) and pJK107 (lanes 3,4) were resolved by electrophoresis on a 1.5\% agaroseformaldehyde gel. Northern blot analysis was performed using the DIG-labeled crr probe. RNA bands corresponding to the full-length crp-crr and truncated $c r r$ mRNAs are indicated by arrowheads. (Lane M) RNA size markers. adds a short peptide tag to incomplete or aberrant polypeptides for degradation by cellular proteases. In addition to the aberrant polypeptides, full-length normal proteins can be tagged by the tmRNA system depending on the C-terminal sequence of the nascent peptide (Hayes et al. 2002a; Sunohara et al. 2002). The commonly accepted view of tmRNA action at stop codons is that tmRNA could compete with the translation termination process (Collier et al. 2002; Hayes et al. 2002a,b; Sunohara et al. 2002). The present study on the model crp genes has led us to propose that the tagging at the stop codon is caused by generation of nonstop mRNA lacking a stop codon. This conclusion is drawn from the following observations: (1) The truncated crp mRNA corresponding to a cleavage product was detected in the absence of tmRNA along with the full-length crp mRNA when the tagging of CRP occurs (Figs. 5, 7); (2) the truncated $\operatorname{crp}$ mRNA was no longer observed in the presence of tmRNA (Figs. 5, 7); (3) the major 3' -ends of the truncated $\operatorname{crp}$ mRNA were mapped at the stop codon (Fig. 4); and (4) the downstream cleavage product, crr mRNA, was detected when the $c r p-c r r$ model fusion gene was analyzed (Fig. 7). Based on these observations, we propose the following scenario for the tmRNA-mediated trans-translation at stop codons (Fig. 9). The particular C-terminal sequences of the nascent peptide somehow interfere with the action of RFs at stop codons, leading to ribosome stalling. The stalled ribosome induces endonucleolytic cleavages of an mRNA around the stop codon, resulting in truncated $\operatorname{crp}$ mRNAs lacking a stop codon. The truncated $\operatorname{crp}$ mRNAs are "normally" recognized by the tmRNA system, resulting in tagging of CRP and ribosome release. The truncated crp mRNAs released from the stalled ribosome by tmRNA are rapidly degraded by exonucleases.

The bacterial toxin RelE was expected to be responsible for the generation of nonstop mRNA in response to a stalled ribosome because RelE was shown to induce endonucleolytic cleavages of mRNAs bound to ribosomes at specific codons including stop codons (Pedersen et al. 2003). However, this is not the case because the nonstop mRNA was normally generated in cells lacking RelE. It should be noted that two other toxins, ChpAK/MazF and ChpBK, have been shown to cleave mRNA at the A-site of the stalled ribosome (Christensen et al. 2003). It is certainly interesting to examine whether these bacterial toxins are involved in endonucleolytic cleavage of mRNA around stop codons in response to a stalled ribosome either alone or in combination. In this respect, a similar cleavage of mRNA in response to ribosome stalling at stop codons was found in $y b e L$ mRNA by Hayes and Sauer (2003). They showed that the cleavage of $y b e L$ mRNA does not require ppGpp, RNaseR, or the bacterial toxins RelE, YoeB, YafQ, MazF, and ChpBK. We also observed that none of the known major endoribonucleases such as RNase E, RNase G, and RNase III are required for the generation of nonstop mRNA (data not shown). Further studies are needed to specify the endo- 


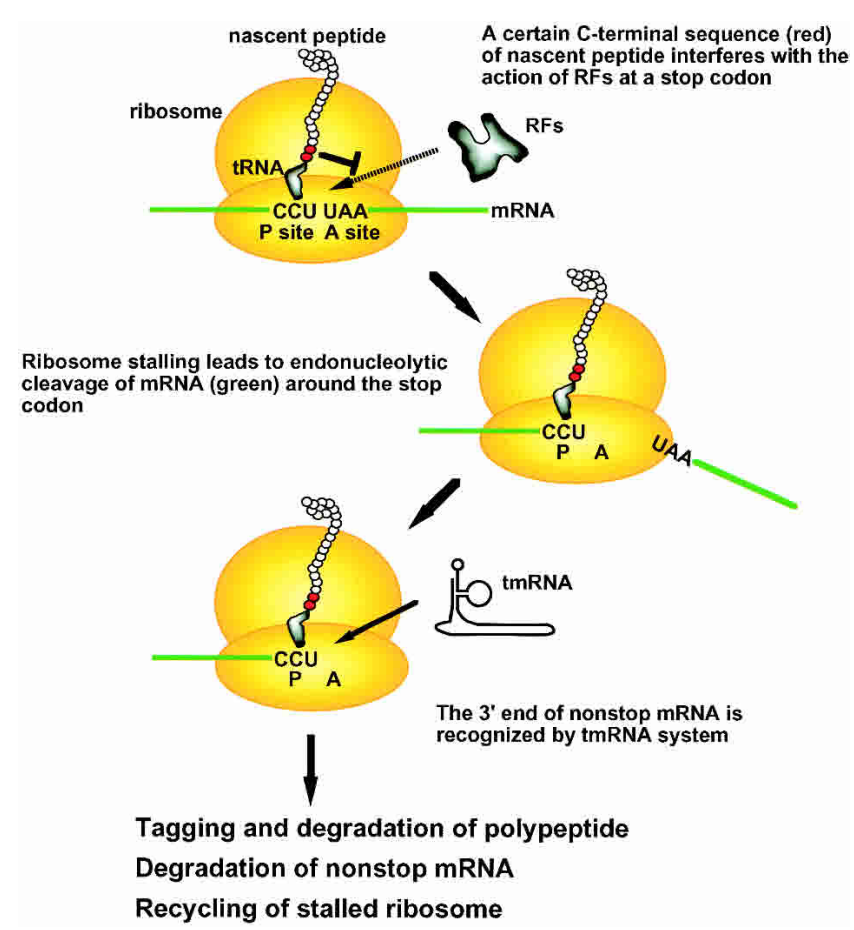

FIGURE 9. Model for mRNA cleavage at a stop codon induced by ribosome stalling.

nuclease activity that cleaves mRNA in response to a stalled ribosome. An attractive possibility would be that the ribosomal RNA and/or proteins might be directly responsible for the endonucleolytic activity that is manifested depending on ribosome stalling. In this connection, it is interesting to note that the ribosome A-site consists largely of RNA (Carter et al. 2000) and that ribosomal protein S16 has a DNA endonuclease activity (Oberto et al. 1996).

Is the endonucleolytic cleavage of mRNA caused by ribosome stalling specific at stop codons? There are two examples in which ribosome stalling seems to be involved in endonucleolytic cleavage of mRNA at specific sites. First, the cleavage of mRNA at a specific site was shown for the processing of fimbrial mRNA encoded by the daa operon in Escherichia coli (Loomis et al. 2001). Interaction of the nascent tripeptide of the DaaP polypeptide with the ribosome appears to cause ribosome stalling, resulting in cleavage of the associated mRNA at a fixed distance upstream. Another example is the cleavage of erm $C$ mRNA coding for a ribosomal RNA methyltransferase that renders the ribosome resistant to erythromycin binding in Bacillus subtilis (Drider et al. 2002). The presence of erythromycin induces ribosome stalling near the $5^{\prime}$-end of ermC mRNA, depending on the particular sequence of the nascent peptide. As a result, the erm C mRNA is cleaved to allow the high-level of translation of methyltransferase presumably by opening of the leader structure. The endonuclease activity responsible for the mRNA cleavage is also not known yet in both cases. It will also be interesting to examine whether or not mRNA cleavage occurs or not at a run of rare arginine codons, where the tagging occurs presumably because of ribosome stalling caused by the deficiency of cognate aminoacyltRNAs (Roche and Sauer 1999). Our preliminary analysis indicates that the endonucleolytic cleavage of mRNA indeed occurs at a run of AGG rare arginine codons, resulting in truncated mRNA lacking a stop codon.

In addition, there are several cases in which specific nascent peptides affect both translation elongation and termination, resulting in ribosome stalling. For example, it is known that the 24-residue product of the tnaC gene prevents the release of the peptide at the stop codon, depending on the availability of tryptophan to regulate the expression of the downstream tna operon (Gong and Yanofsky 2002). Another example is translation arrest in the chloramphenicol transacetylase gene (cat) in Gram-positive bacteria by the nascent pentapeptide (MVKTD), which interacts with the ribosome in the presence of chloramphenicol (Lovett and Rogers 1996). More recently, it has been shown that a specific nascent peptide in the $\sec M$ gene induces translation arrest, presumably by interacting with the ribosomal exit tunnel (Nakatogawa and Ito 2002). It will certainly be interesting to study whether ribosome stalling leads to mRNA cleavage and tmRNA-mediated protein tagging as well as mRNA degradation in these cases.

\section{MATERIALS AND METHODS}

\section{Media and growth conditions}

Cells were grown aerobically at $37^{\circ} \mathrm{C}$ in Luria-Bertani (LB) medium (Miller 1972). Antibiotics were used at the following concentrations: ampicillin $(50 \mu \mathrm{g} / \mathrm{mL})$ and chloramphenicol $(30 \mu \mathrm{g} /$ $\mathrm{mL}$ ). Bacterial growth was monitored by determining the optical density at $600 \mathrm{~nm}$.

\section{Strains and plasmids}

The E. coli K-12 strains used are TA341 (W3110 $\Delta$ crp), TA501

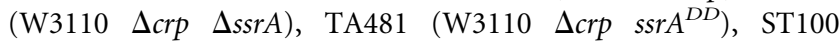
(W3110 $\Delta$ relEB), ST101 (W3110 $\Delta s s r A \Delta$ relEB), and ST102 (W3110 ssr $A^{D D} \Delta$ relEB). The gene knockout system of Datsenko and Wanner (2000) was used to construct the strains. All plasmids that express wild-type and variant forms of CRP are derived from pHA7 carrying the crp gene under the bla promoter (Aiba et al. 1982). Plasmid pHA7M expressing wild-type CRP was described previously (Abo et al. 2002). Plasmids pJK021, pJKA327, pJK101, pJK102, and their derivatives expressing variant forms of CRP were constructed from $\mathrm{pHA7M}$ by PCR mutagenesis using appropriate primers. Plasmids pST602 and pJK107 carrying the crp-crr fusion genes were constructed from pST513 (Abo et al. 2002) by PCR mutagenesis using appropriate primers.

\section{Western blotting}

Bacterial cells were grown in LB medium containing appropriate antibiotics to mid-log phase. Culture samples $(1 \mathrm{~mL})$ were cen- 
trifuged, and the pellets were suspended in $50 \mu \mathrm{L}$ of $\mathrm{H}_{2} \mathrm{O}$. The cell suspensions were mixed with $50 \mu \mathrm{L}$ of $2 \times$ loading buffer ( $4 \%$ SDS, $10 \%$ 2-mercaptoethanol, $125 \mathrm{mM}$ Tris- $\mathrm{HCl}$ at $\mathrm{pH} 6.8,10 \%$ glycerol, $0.2 \%$ bromophenol blue) and heated for $5 \mathrm{~min}$ at $100^{\circ} \mathrm{C}$. For Western blotting, the total extracts of the indicated amounts were subjected to a $0.1 \%$ SDS, $12 \%$ or $15 \%$ PAGE and transferred to Immobilon membrane (Millipore). The membrane was probed with anti-CRP antibodies using the ECL system (Amersham Life Science).

\section{Mass spectrometry}

The untagged and tagged CRP-GP proteins were purified from TA481 (W3110 $\Delta$ crp ssr $A^{D D}$ ) cells carrying pJK021 according to the conventional procedure (Eilen et al. 1978). For mass spectrometry (MS) analysis, the purified untagged and DD-tagged proteins were separated by $12 \%$ SDS-PAGE. The bands were cut out from the gel, and a small piece of each band containing $\sim 0.5 \mu \mathrm{g}$ of protein was treated with $0.1 \mu \mathrm{g}$ of lysyl endopeptidase (Wako) in $20 \mu \mathrm{L}$ of $100 \mathrm{mM}$ Tris- $\mathrm{HCl}(\mathrm{pH} 9.0)$ for $12 \mathrm{~h}$ at $37^{\circ} \mathrm{C}$. The digested peptides were eluted with $300 \mu \mathrm{L}$ of $50 \%$ acetonitrile, $5 \%$ formic acid, and concentrated to $20 \mu \mathrm{L}$. Then, the sample was desalted with a zip-tip reverse-phase column, mixed with $1 \% \alpha$-CHCA $(\alpha$-cyano-4-hydroxycinnamic acid) in $70 \%$ acetonitrile, and subjected to MALDI/TOF-MS.

\section{RNA analyses}

Total RNA was isolated from cells grown to mid-log phase as described (Aiba et al. 1981). The total RNA was resolved by either $2.0 \%$ or $1.5 \%$ agarose-gel electrophoresis in the presence of formaldehyde and blotted onto a Hybond- $\mathrm{N}^{+}$membrane (Amersham) as described. The mRNAs were visualized using digoxigenin (DIG) reagents and kits for nonradioactive nucleic acid labeling and detection system (Roche) according to the procedure specified by the manufacturer. The DIG-labeled DNA probes used were 576-bp probe A corresponding to the crp coding region and 507-bp probe $\mathrm{B}$ corresponding to the $\mathrm{cr}$ coding region. The DIG-labeled RNA marker III (Roche) was used to estimate the size of RNA bands. The 3 '-ends of $\operatorname{crp}$ mRNA were determined by $\mathrm{S} 1$ nuclease assay as described (Aiba et al. 1981). A DNA fragment corresponding to the 3 '-region of crp mRNA was prepared by PCR from pJK021 encoding CRP-GP and digested with Sau3AI that is located $50 \mathrm{bp}$ upstream of the $\operatorname{crp}$ stop codon. The Sau3AI $3^{\prime}$-end of the resulting 188-bp fragment was labeled with $\left[\alpha-{ }^{32} \mathrm{P}\right] \mathrm{dGTP}$ by Klenow enzyme. The ${ }^{32} \mathrm{P}$-labeled fragment was used as a DNA probe (probe C) for S1 assay. The DNA probe and total RNA were hybridized and treated with increasing amounts $\mathrm{S} 1$ nuclease for $15 \mathrm{~min}$ at $37^{\circ} \mathrm{C}$. The resulting products were analyzed on an $8 \%$ or polyacrylamide- $8 \mathrm{M}$ urea gel. The ends of mRNAs were identified by using the Maxam-Gilbert A $+\mathrm{G}$ ladder of the DNA probes as reference.

\section{ACKNOWLEDGMENTS}

We thank Dr. Robert Zimmermann for editorial suggestions and for careful reading of the manuscript. This work was supported by Grants-in-Aid from the Ministry of Education, Culture, Sports, Science and Technology of Japan.
The publication costs of this article were defrayed in part by payment of page charges. This article must therefore be hereby marked "advertisement" in accordance with 18 USC section 1734 solely to indicate this fact.

Received August 26, 2003; accepted November 6, 2003.

\section{REFERENCES}

Abe, H., Abo, T., and Aiba, H. 1999. Regulation of intrinsic terminator by translation in Escherichia coli: Transcription termination at a distance downstream. Genes Cells 4: 87-97.

Abo, T., Inada, T., Ogawa, K., and Aiba, H. 2000. SsrA-mediated tagging and proteolysis of LacI and its role in the regulation of lac operon. EMBO J. 19: 3762-3769.

Abo, T., Ueda, K., Sunohara, T., Ogawa, K., and Aiba, H. 2002. SsrAmediated protein tagging in the presence of miscoding drugs and its physiological role in Escherichia coli. Genes Cells 7: 629-638.

Aiba, H., Adhya, S., and de Crombrugghe, B. 1981. Evidence for two functional gal promoters in intact Escherichia coli cells. J. Biol. Chem. 256: 11905-11910.

Aiba, H., Fujimoto, S., and Ozaki, N. 1982. Molecular cloning and nucleotide sequencing of the gene for E. coli cAMP receptor protein. Nucleic Acids Res. 10: 1345-1361.

Carter, A.P., Clemons, W.M., Brodersen, D.E., Morgan-Warren, R.J., Wimberly, B.T., and Ramakrishnan, V. 2000. Functional insights from the structure of the $30 \mathrm{~S}$ ribosomal subunit and its interactions with antibiotics. Nature 407: 340-348.

Christensen, S.K. and Gerdes, K. 2003. RelE toxins from Bacteria and Archaea cleave mRNAs on translating ribosomes, which are rescued by tmRNA. Mol. Microbiol. 48: 1389-1400.

Christensen, S.K., Pedersen, K., Hansen, F.G., and Gerdes, K. 2003. Toxin-antitoxin loci as stress-response-elements: ChpAK/MazF and ChpBK cleave translated RNAs and are counteracted by tmRNA. J. Mol. Biol. 332: 809-819.

Collier, J., Binet, E., and Bouloc, P. 2002. Competition between SsrA tagging and translational termination at weak stop codons in Escherichia coli. Mol. Microbiol. 45: 745-754.

Datsenko, K.A. and Wanner, B.L. 2000. One-step inactivation of chromosomal genes in Escherichia coli K-12 using PCR products. Proc. Natl. Acad. Sci. 97: 6640-6645.

Drider, D., DiChiara, J.M., Wei, J., Sharp, J.S., and Bechhofer, D.H. 2002. Endonuclease cleavage of messenger RNA in Bacillus subtilis. Mol. Microbiol. 43: 1319-1329.

Eilen, E., Pampeno, C., and Krakow, J.S. 1978. Production and properties of the $\alpha$ core derived from the cyclic adenosine monophosphate receptor protein of Escherichia coli. Biochemistry 17: 24692473.

Gong, F. and Yanofsky, C. 2002. Instruction of translating ribosome by nascent peptide. Science 297: 1864-1867.

Hayes, C.S. and Sauer, R.T. 2003. Cleavage of the A site mRNA codon during ribosome pausing provides a mechanism for translational quality control. Mol. Cell 12: 903-911.

Hayes, C.S., Bose, B., and Sauer, R.T. 2002a. Proline residues at the C-terminus of nascent chains induce SsrA-tagging during translation termination. J. Biol. Chem. 277: 33825-33832.

- 2002b. Stop codons preceded by rare arginine codons are efficient determinants of SsrA tagging in Escherichia coli. Proc. Natl. Acad. Sci. 99: 3440-3445.

Karzai, A.W., Roche, E.D., and Sauer, R.T. 2000. The SsrA-SmpB system for protein tagging, directed degradation and ribosome rescue. Nat. Struct. Biol. 7: 449-455.

Keiler, K.C., Waller, P.R., and Sauer, R.T. 1996 Role of a peptide tagging system in degradation of proteins synthesized from damaged messenger RNA. Science 271: 990-993.

Loomis, W.P., Koo, J.T., Cheung, T.P., and Moseley, S.L. 2001. A 


\section{Sunohara et al.}

tripeptide sequence within the nascent DaaP protein is required for mRNA processing of a fimbrial operon in Escherichia coli. Mol. Microbiol. 39: 693-707.

Lovett, P.S. and Rogers, E.J. 1996 Ribosome regulation by the nascent peptide. Microbiol. Rev. 60: 366-385.

Miller, J. 1972. Experiments in molecular genetics. Cold Spring Harbor Laboratory, Cold Spring Harbor, NY.

Nakatogawa, H. and Ito, K. 2002. The ribosomal exit tunnel functions as a discriminating gate. Cell 108: 629-636.

Oberto, J., Bonnefoy, E., Mouray, E., Pellegrini, O., Wikstrom, P.M., and Rouviere-Yaniv, J. 1996 The Escherichia coli ribosomal protein S16 is an endonuclease. Mol. Microbiol. 19: 1319-1330.

Pedersen, K., Zavialov, A.V., Pavlov, M.Y., Elf, J., Gerdes, K., and Ehrenberg, M. 2003. The bacterial toxin RelE displays codon-spe- cific cleavage of mRNAs in the ribosomal A site. Cell 112: 131-140. Roche, E.D. and Sauer, R.T. 1999. SsrA-mediated peptide tagging caused by rare codons and tRNA scarcity. EMBO J. 18: 4579-4589.

Sunohara, T., Abo, T., Inada, T., and Aiba, H. 2002. The C-terminal amino acid sequence of nascent peptide is a major determinant of SsrA tagging at all three stop codons. RNA 8: 1416-1427.

Ueda, K., Yamamoto, Y., Ogawa, K., Abo, T., Inokuchi, H., and Aiba, H. 2002. Bacterial SsrA system plays a role in coping with unwanted translational readthrough caused by suppressor tRNAs. Genes Cells 7: 509-519.

Yamamoto, Y., Sunohara, T., Jojima, K., Inada, T., and Aiba, H. 2003. SsrA-mediated trans-translation plays a role in mRNA quality control by facilitating degradation of truncated mRNAs. RNA 9: 408418 . 

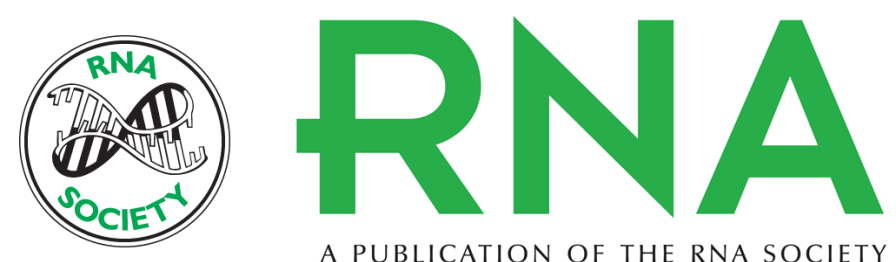

A PUBLICATION OF THE RNA SOCIETY

\section{Nascent-peptide-mediated ribosome stalling at a stop codon induces mRNA cleavage resulting in nonstop $m R N A$ that is recognized by tmRNA}

TAKAFUMI SUNOHARA, KAORU JOJIMA, YASUFUMI YAMAMOTO, et al.

RNA 2004 10: 378-386

References This article cites 25 articles, 10 of which can be accessed free at:

http://rnajournal.cshlp.org/content/10/3/378.full.html\#ref-list-1

License

Email Alerting Receive free email alerts when new articles cite this article - sign up in the box at the Service top right corner of the article or click here. 